\title{
ANFIBIOS Y REPTILES ASOCIADOS A CINCO COBERTURAS DE LA TIERRA, MUNICIPIO DE DIBULLA, LA GUAJIRA, COLOMBIA
}

\author{
JuLIo CÉSAR ACUÑA-VARGAS
}

\author{
Universidad de La Guajira, Grupo de investigación en Ecología, Biodiversidad en Ecosistemas Tropicales \\ (Ebet), Facultad de Ciencias Básicas y Aplicadas. Riohacha, La Guajira - Colombia. Kilómetro 5 Vía a Maicao, \\ Laboratorio de Ciencias Biológicas, Riohacha, La Guajira - Colombia. \\ <jcacuna@uniguajira.edu.co>; <julioacunavargas@gmail.com>; <ebet.ecosistemas.tropicales@gmail.com> \\ Recibido: 13/03/2015; aceptado: 07/03/2016
}

Acuña-Vargas, J. C. 2016. Anfibios y Reptiles asociados a cinco coberturas de la tierra, municipio de Dibulla, La Guajira, Colombia. Acta Zoológica Mexicana (n. s.), 32(2): 133-146.

RESUMEN. Se desarrolló un estudio para determinar la composición de anfibios y reptiles del área rural del municipio de Dibulla, La Guajira, asociados a cinco coberturas de la tierra con diferentes grados de perturbación. Se realizaron 40 muestreos entre marzo y octubre de 2012, empleando técnicas estandarizadas para monitorear anfibios y reptiles (VES y Transecto de banda auditiva), sobre las coberturas de la tierra identificadas y seleccionadas. En un área de estudio de 20 $\mathrm{km}^{2}$, se registraron 22 especies de anfibios y 40 especies de reptiles, las cuales representan entre 31 y 50\% de las especies registradas para la región Caribe y el Bs-t en Colombia. La cobertura Tejido urbano discontinuo (ZU), fue la cobertura que presentó mayor riqueza de especies (21 anfibios y 33 reptiles) y mayor abundancia proporcional $\left(H^{\prime}=2.83 ; H^{\prime} \max =3.98 ; J^{\prime}=0.71\right)$; los valores de equidad $\left(J^{\prime}\right)$ se presentaron en el rango de 0.66-0.76 entre las diferentes coberturas de la tierra. No existen diferencias significativas en la diversidad $\left(H^{\prime}\right)$ entre la cobertura ZU y la cobertura Bosque fragmentado inundable con vegetación secundaria (Bfi) $\left(t_{0.05(2) 18198246.5}=1.96\right)$; la cobertura Cultivo de palma (CP) y la cobertura Mosaico de pastos y cultivos transitorios inundables con setos de árboles y arbustos (MPC) $\left(t_{0.05(2) 7370.49}=\right.$ 1.96). Las coberturas de la tierra que presentaron mayor relación $\left(I_{J}\right.$ $=0.68$ ) fueron la asociación CP y Mosaico de Pastos enmalezados o enrastrojados con setos de árboles y arbustos (MP), mientras que la relación más baja la presentó la asociación Bfi y MP $\left(I_{J}=0.55\right)$. Los reptiles fueron el grupo más diverso y los anfibios registraron los mayores valores de abundancia durante el estudio. Éste es el primer aporte referente a la composición de la herpetofauna para esta localidad; la riqueza de anfibios y reptiles puede considerarse alta en relación con otros estudios realizados en el departamento de La Guajira, aunque la composición de anfibios y reptiles que presentaron mayor abundancia se registran como especies generalistas de amplio rango de distribución y que pueden desarrollarse en ambientes con diferentes grados de perturbación, lo cual evidencia la necesidad de estudios dirigidos a evaluar la situación de la biodiversidad en esta localidad con otros enfoques que permitan generar una interpretación más cercana al impacto de los usos del suelo sobre la coberturas de la tierra y la diversidad asociada, sin omitir cambios más complejos como la homogeneización biótica.

Palabras claves: Biodiversidad, anfibios, reptiles, coberturas de la tierra, usos del suelo.
Acuña-Vargas, J. C. 2016. Amphibians and Reptiles associated to five land coverages, municipality of Dibulla, La Guajira, Colombia. Acta Zoológica Mexicana (n. s.), 32(2): 133-146.

ABSTRACT. This study was conducted to determine the composition and structure of amphibians and reptiles of the rural municipality of Dibulla, La Guajira, associated to five land cover with different degrees of disturbance. Forty samples were made between March and October 2012 using standardized techniques for monitoring amphibians and reptiles (VES and audio strip transect), on the coverage of the identified and selected land. A total of 22 species of amphibians and 40 species of reptiles were recorded in the study area of $20 \mathrm{~km}^{2}$, representing between 31 and $50 \%$ of the species recorded for the Caribbean and Bs-t in Colombia. The coverage discontinuous urban fabric (ZU), contained higher species richness (21 amphibians and 33 reptiles) and greater proportional abundance $\left(H^{\prime}=2.83 ; H^{\prime} \max =3.98 ; J^{\prime}=0.71\right)$; the values of equity $\left(J^{\prime}\right)$ occurred in the range of 0.66 to 0.76 between different land coverages. No significant differences in diversity $\left(H^{\prime}\right)$ between the ZU coverage and fragmented floodplain forest with secondary vegetation (Bfi) $\left(t_{0.05(2) 18198246.5}=1.96\right)$; Cultivation palm coverage (CP) and Mosaic flood grasses and temporal crops with hedges of trees and shrubs (MPC) $\left(t_{0.05(2) 7370.49}=1.96\right)$. The land coverages that had a close relationship $\left(I_{J}=0.68\right)$ were the $\mathrm{CP}$ association and mosaic weedy grasses with hedges of trees and shrubs (MP), while the lowest ratio was the association Bfi and MP $\left(I_{J}=0.55\right)$. The reptiles were the most diverse group and amphibians recorded the highest abundance values during the study. The present study is the first contribution concerning the composition of the herpetofauna for this location; the richness of amphibians and reptiles can be considered high in relation to other studies in La Guajira, although the composition of amphibians and reptiles showing the greatest abundance are recorded as wide distribution species and tolerant to environments with different degrees of disturbance, and are considered generalists. This shows the need for studies aimed at assessing the status of biodiversity in this locality with other approaches for generate a closer understanding of the impact of land use on land cover and diversity associated, without omitting more complex changes as biotic homogenization.

Key words: Biodiversity, amphibians, reptiles, land cover, land use. 


\section{INTRODUCCIÓN}

El bosque seco tropical (Bs-t) en Colombia lo representan pequeñas áreas en paisajes fragmentados resultado de labores agrícolas traducidas en deforestación de coberturas naturales de este ecosistema (Pizano et al. 2014), lo que resulta en una gran limitante para el desarrollo de la riqueza de especies de grupos como anfibios y reptiles por la baja conectividad entre fragmentos (Urbina-Cardona et al. 2014) y su alta sensibilidad a cambios ambientales que generan la fragmentación de hábitat (Duellman \& Trueb 1986; Whiles et al. 2006; Almeida-Gomes \& Rocha 2014). Estas interrupciones generadas por la conversión del bosque en paisajes antropogénicos brindan limitadas opciones para el desarrollo de anfibios y reptiles, generando paisajes homogéneos, reduciendo la complejidad de los hábitat, la posibilidad de diversos microhábitat y mayores ofertas ambientales que favorezcan el aumento de la riqueza de estos grupos (Almeida-Gomes \& Rocha 2014), convirtiéndose en uno de los principales factores del declive y extinción de especies (Zank et al. 2014).

La región Caribe Colombiana presenta 97.6\% de su área natural intervenida, aun así, la representación del bosque seco tropical en la región Caribe presenta los relictos de bosque en mejor estado de conservación (Pizano et al. 2014), hoy día amenazados por la expansión de la frontera agrícola (García-Oliva \& Jaramillo 2011). La fragmentación de hábitat genera cambios sobre las poblaciones de anfibios (Urbina-Cardona et al. 2006; Gardner et al. 2007a; Gardner et al. 2007b; Cáceres-Andrade \& UrbinaCardona 2009; Isaacs \& Urbina-Cardona 2011; Hof et al. 2011) y reptiles (Glor et al. 2001; Urbina-Cardona et al. 2006; Gardner et al. 2007a; Gardner et al. 2007b; Carvajal-Cogollo \& Urbina-Cardona 2008; Urbina-Cardona et al. 2008).

Los estudios sobre la composición y estructura de anfibios y reptiles en el departamento de la Guajira son incipientes y aislados, particularmente en el área del presente estudio, y la información sobre biodiversidad es muy escasa y limitada. Se encuentran fragmentos de bosque de ribera y bosque seco, inmersos en una gran dinámica de modificación de coberturas de la tierra (pastizales, cultivos, carreteras), que amenazan con la disminución y pérdida de los fragmentos de bosque y los cambios que éstos pueden generar en la composición, abundancia y distribución de las poblaciones de anfibios y reptiles.

El presente estudio tiene como objetivo presentar la estructura y composición de las comunidades de anfibios y reptiles presentes en diferentes coberturas de la tierra con diferentes grados de perturbación en el área rural del municipio de Dibulla, Caserío Casa Aluminio y generar conocimiento básico sobre la herpetofauna de este sector que contribuya al desarrollo de iniciativas futuras de conservación en estos grupos y sus hábitat.

\section{MATERIALES Y MÉTODOS}

Área de estudio. La zona de estudio se localiza en el municipio de Dibulla, Vereda Casa Aluminio, departamento de la Guajira, Colombia ( $11^{\circ} 14^{\prime} 16^{\prime \prime}$ y $11^{\circ} 13^{\prime} 27^{\prime \prime}$ N; $73^{\circ}$ $18^{\prime} 49^{\prime \prime}$ y $73^{\circ} 18^{\prime} 25^{\prime \prime} \mathrm{O}$ ), presenta una altitud entre los 3 y 17 msnm (Fig. 1). El clima es cálido, con temperatura promedio anual de $30{ }^{\circ} \mathrm{C}$ y un régimen bimodal de lluvias con una precipitación total anual de aproximadamente $1700 \mathrm{~mm}$.

El estudio se realizó en un área que presenta diferentes coberturas de la tierra, con la presencia de terrenos "artificializados”, agrícolas, bosque y áreas seminaturales, según la metodología CORINE land cover adaptada para Colombia (IDEAM 2010), donde se identificaron cinco diferentes tipos de coberturas de la tierra: cultivo de palma de aceite, mosaico de pastos enmalezados o enrastrojados con setos de árboles y arbustos, mosaico de pastos y cultivos transitorios inundables no arbolado con setos de árboles y arbustos, bosque fragmentado inundable con vegetación secundaria y tejido urbano discontinuo (Cuadro 1).

Cultivo de palma (CP): se refiere a un área con cultivos permanentes arbóreos cuyo ciclo vegetativo es mayor que un año, produciendo varias cosechas sin necesidad de volver a plantar (IDEAM 2010), en el presente estudio se relaciona con un cultivo joven de palma de aceite (Elaeis guineensis) con dos años de siembra y una extensión de 82.9 ha, la vegetación presenta una distribución vertical entre los 0 y $3 \mathrm{~m}$, presenta un sistema de canales de riego artificial en su interior y límites, con senderos creados por la siembra de palma, tapizados por la fabácea Kudzu (Pueraria phaseoloides), el cultivo de palma limita con el fragmento de bosque y el mosaico de pastos enmalezados o enrastrojados.

Mosaico de pastos enmalezados o enrastrojados con setos de árboles y arbustos (MP): Son unidades que reúnen dos o más clases de coberturas, con parcelas pequeñas menores a 25 ha (IDEAM 2010), hacen referencia en el presente estudio a un área cubierta de pastos y arbustos considerados arvenses de cultivos, bordeados por cercas vivas, comprende una extensión de 13.3 ha, la vegetación 


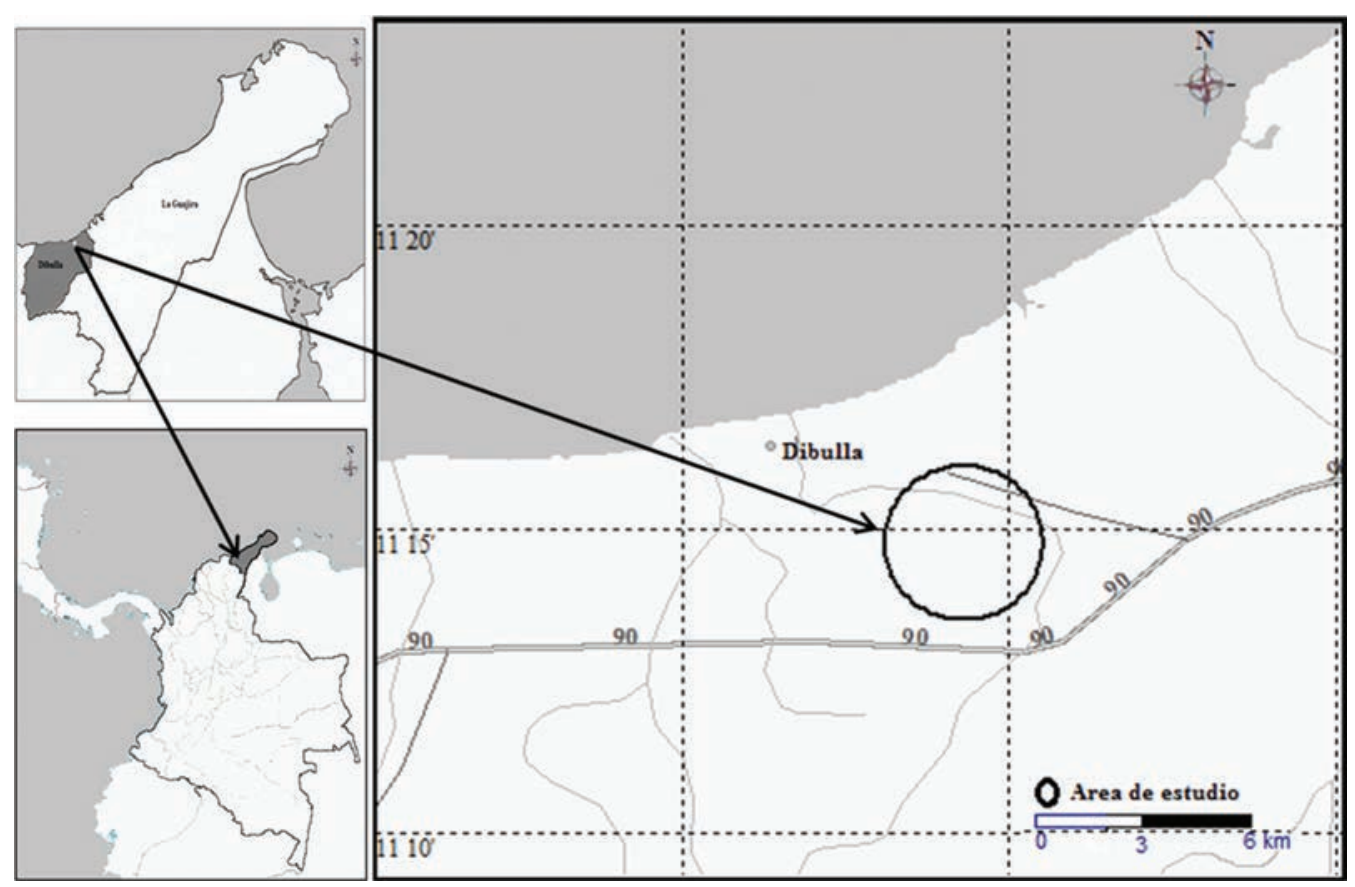

Figura 1. Mapa del área de estudio, municipio de Dibulla, departamento de La Guajira (Colombia).

Cuadro 1. Clasificación coberturas de la tierra según leyenda nacional de coberturas de la tierra, Metodología CORINE land cover adaptada para Colombia (IDEAM 2010).

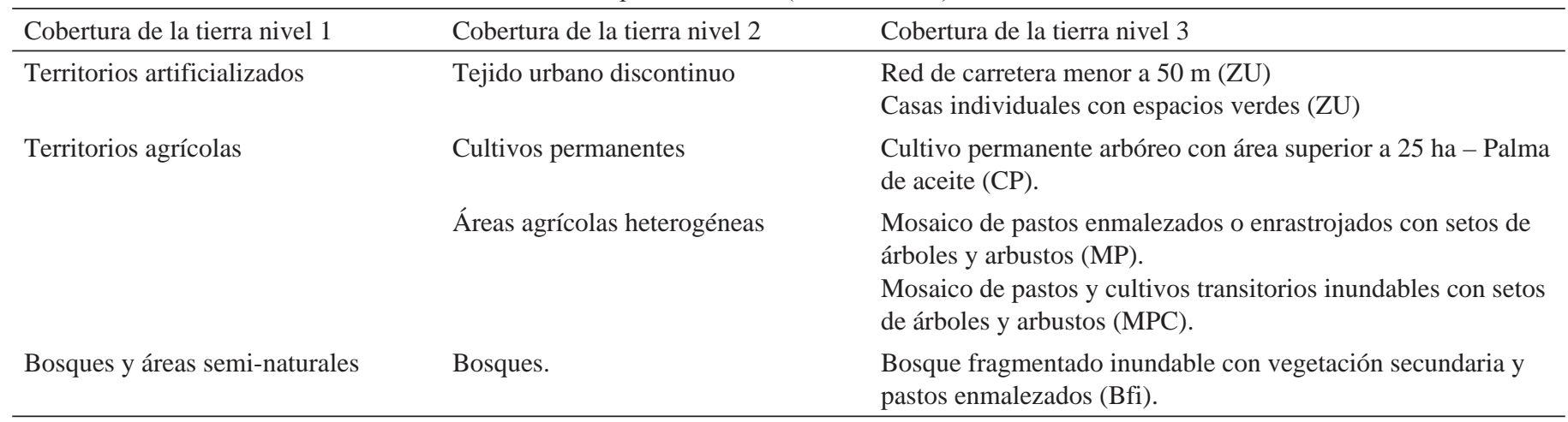

presenta una distribución vertical entre los 0 y $1 \mathrm{~m}$, esta cobertura no presenta canales de riego, parte del suelo se encuentra descubierto, esta área se utiliza para el pastoreo de ganado vacuno y presenta setos de árboles y arbustos con una distribución vertical entre los 0 y $10 \mathrm{~m}$.

Mosaico de pastos y cultivos transitorios inundables con setos de árboles y arbustos (MPC): Son unidades que reúnen dos o más clases de coberturas, con parcelas pequeñas menores a 25 ha (IDEAM 2010), hace referencia a una superficie inundable, con la presencia de pastos y cultivos cuyo ciclo vegetativo es menor que un año, además se encuentra cercado por árboles y arbustos (IDEAM
2010), comprende un área destinada al cultivo de arroz (Oryza sativa), entre otras especies, con una extensión de 15 ha, la vegetación presenta una distribución vertical entre los 0 y 2 m; esta área presenta canales de riego, la superficie de la tierra se encuentra cubierta de vegetación, se utiliza para el pastoreo de ganado vacuno de manera esporádica, posee un seto de árboles y arbustos con una distribución vertical entre los 0 y $10 \mathrm{~m}$.

Bosque fragmentado inundable con vegetación secundaria (Bfi): hace referencia a un fragmento de bosque seco tropical, con vegetación secundaria (IDEAM 2010), con una extensión de 49.4 ha, el cual presenta una dis- 
tribución vertical de la vegetación entre los 0-25 m de altura.

Tejido urbano discontinuo (ZU): Hace referencia a espacios, viviendas y vías que cubren la superficie de la tierra de manera dispersa y discontinua, ya que el resto del área está cubierto por vegetación (IDEAM 2010), comprende una superficie de 18 ha, la vegetación presenta una distribución vertical entre los 0 y $20 \mathrm{~m}$; esta cobertura limita con todas las coberturas de la tierra descritas en el presente estudio.

Trabajo de campo. Entre los meses de marzo y octubre del 2012 se realizaron monitoreos periódicos al área de estudio, los cuales consistieron en cinco visitas por mes, para estudiar la estructura y composición de anfibios y reptiles asociados a las cinco coberturas de la tierra descritas.

Se utilizó la técnica estandarizada de relevamientos por encuentros visuales (VES), para anfibios (Heyer et al. 1994) y reptiles (McDiarmid et al. 2012) sobre caminatas aleatorias, y se combinó para el caso de anfibios con la técnica de transectos de bandas auditivas (Heyer et al. 1994), se realizaron dos recorridos con longitud de $500 \mathrm{~m}$ cada uno, y ancho aproximado de $10 \mathrm{~m}$ para cada tipo de cobertura de la tierra. Los muestreos diurnos se realizaron entre las 8:00 y 17:00, los muestreos nocturnos entre las 18:00 y 3:00; se seleccionaron seis horas de estudio en cada periodo de muestreo, con el fin de obtener el mayor horario de actividad para anfibios y reptiles.

Se registraron datos ambientales (temperatura, humedad, luminosidad, punto de rocío), por medio de data loggers (Onset Hobo) con lecturas ambientales programadas a intervalos de un minuto en cada cobertura de la tierra, además del uso de un termohigrómetro digital (Halthen) y un luxómetro digital (PCE-174) para la toma de lecturas ambientales en el momento de la captura para los grupos de estudio. Se tomaron registros comportamentales, representados en la actividad exhibida por los individuos observados, en el momento de la captura, además se georreferenciaron y fotografiaron, según la posibilidad.

Se colectó un voucher de dos ejemplares por especie por cobertura de la tierra los cuales fueron depositados en la colección biológica de la Universidad de La Guajira (en proceso de inscripción ante el Instituto de Investigación de Recursos Biológicos, Alexander von Humboldt). La determinación taxonómica se realizó siguiendo los criterios descritos en literatura especializada (Campbell \& Lamar 1989; Roze 1996; Narvaes \& Rodrigues 2009; Ugueto \& Harvey 2011; Hedges \& Conn 2012; Mccraine \& Hedges 2013; Acosta-Galvis 2015) para cada uno de los dos grupos de vertebrados.

\section{ANÁLISIS DE DATOS}

Diversidad alfa. Para la medida de la diversidad alfa se realizó la medición de la riqueza de especies en cada cobertura de la tierra (Moreno 2001), además se utilizaron métodos basados en la medición de la estructura de la comunidad (Moreno 2001), para ésta se realizaron curvas de acumulación de especies para estimar la precisión del esfuerzo de muestreo, por medio del estimador de estructura no paramétrico Chao 1, basado en el registro de especies raras de la muestra (Moreno 2001; Magurran 2004), considerado un estimador de riqueza riguroso y por medio de índices de abundancia proporcional (Moreno 2001), utilizando el índice de Pielou ( $J$ ') para cuantificar la contribución de la equidad a la diversidad total encontrada y el índice de Shannon-Wiener $\left(H^{\prime}\right)$ para medir la diversidad y uniformidad (Moreno 2001). Para probar la hipótesis nula que la diversidad $\left(H^{\prime}\right)$ de las cinco coberturas son iguales, se calculó el índice de diversidad ponderado $(\mathrm{Hp})$ siguiendo a Hutchenson (1970) descrito en Zar (2010) y calculando la varianza del índice de diversidad ponderado para cada cobertura y aplicando un test de $t$ (Moreno 2001).

Para la medida de la abundancia relativa de anfibios y reptiles en cada cobertura de la tierra, se tuvo en cuenta el número de individuos registrados por especie en relación con el número total de individuos registrados para cada cobertura de la tierra identificada. Se realizaron curvas de Whittaker o de rango-abundancia (Magurran 2004), de forma general para el área de estudio y para cada grupo (anfibios y reptiles) en cada cobertura de la tierra identificada.

Diversidad beta. Para la medida de la diversidad beta se utilizó el índice de similitud-disimilitud de Jaccard $\left(I_{J}\right)$ que tiene en cuenta las especies compartidas con las exclusivas y se realizó el análisis de complementariedad $\left(C_{\mathrm{AB}}\right)$ de Colwell \& Coddington (Moreno 2001).

\section{RESULTADOS}

\section{Diversidad alfa}

Composición. Se obtuvieron 14,347 registros de la herpetofauna en el área de estudio, pertenecientes a 4 órdenes, 22 familias, 48 géneros y 62 especies de anfibios y reptiles. Los reptiles presentaron mayor diversidad con 3 órdenes, 15 familias, 35 géneros y 40 especies, siendo la familia 
Colubridae la que presentó mayor aporte a los registros de riqueza con 15 géneros y 17 especies (Cuadro 2).

La cobertura de la tierra $\mathrm{ZU}$, fue la que presentó mayor riqueza de especies ( $S=54 ; 21$ anfibios y 33 reptiles), seguido de la cobertura MPC $(S=42 ; 14$ anfibios y 28 reptiles); $\mathrm{CP}(S=39 ; 15$ anfibios y 24 reptiles) y Bfi ( $S=$ $39 ; 14$ anfibios y 25 reptiles); MP ( $S=37 ; 17$ anfibios y 20 reptiles) (Cuadro 2$)$.

Estructura. Al realizar el análisis de la curva de acumulación se registra que el esfuerzo de muestreo para cada cobertura de la tierra estuvo representado entre el $85.51 \%$ de las especies previstas por el estimador, indicando un esfuerzo de muestreo representativo (Fig. 2).

En relación con el uso de índices de abundancia proporcional, la cobertura de la tierra que presentó mayor diversidad corresponde a ZU $\left(S=54 ; H^{\prime}=2.83 ; H^{\prime}\right.$ max $\left.=3.98 ; J^{\prime}=0.71\right)$, seguido de Bfi $\left(S=39 ; H^{\prime}=2.80 ; H^{\prime}\right.$ $\left.\max =3.66 ; J^{\prime}=0.76\right)$; para todos los análisis, los valores de equidad ( $\left.J^{\prime}\right)$ se presentaron en el rango de $0.66 \mathrm{y}$ 0.76 indicando que no se presentaron especies altamente dominantes en las coberturas de la tierra estudiadas, presentando estos valores una distribución relativamente homogénea (Cuadro 3).

No existen diferencias significativas en la diversidad $\left(H^{\prime}\right)$ entre las coberturas ZU y Bfi $\left(t_{0.05(2) 18198246.5}=1.96\right) \mathrm{y}$ CP y MPC $\left(t_{0.05(2) 7370.49}=1.96\right)$ las cuales presentaron valores $t$ menores de los valores $t$ de las tablas $(0.67$ para ZU y Bfi; 1.88 para CP y MPC), se acepta la hipótesis nula y se concluye que la diversidad de anfibios y reptiles para estas coberturas es igual; las demás posibles relaciones entre las coberturas de la tierra estudiadas presentaron valores $t$ mayores de los valores $t$ de las tablas, por lo cual se rechaza la hipótesis nula.

Abundancia relativa. Los anfibios registraron los mayores valores de abundancia durante el estudio, las especies que presentaron mayor abundancia relativa fueron Leptodactylus insularum (0.165), Leptodactylus fragilis $(0.127) \mathrm{y}$ Rhinella granulosa (0.083); en el caso de los reptiles estos valores los presentan las especies Cnemidophorus lemniscatus (0.079), Anolis auratus (0.078) y Ameiva bifrontata (0.044) (Cuadro 2).

Los mayores registros de abundancia relativa para los anfibios por cobertura de la tierra en orden de extensión de terreno de mayor a menor fueron, en CP, $L$. fragilis (0.17), L. insularum (0.125), R. granulosa (0.105); en Bfi, L. fragilis (0.184), Leptodactylus poecilochilus (0.134), Leptodactylus fuscus (0.074); en MPC, L. insularum (0.242), Dendropsophus microcephalus (0.172), L. fragilis (0.090); en MP, L. fragilis (0.157), L. insularum (0.138), D. microcephalus (0.117) y para ZU, L. insularum (0.219), R. granulosa (0.133), L. fragilis (0.068), siendo esta última la cobertura de la tierra que registró mayor riqueza de especies (21) durante el estudio (Cuadro 2). Para los reptiles los valores de abundancia relativa

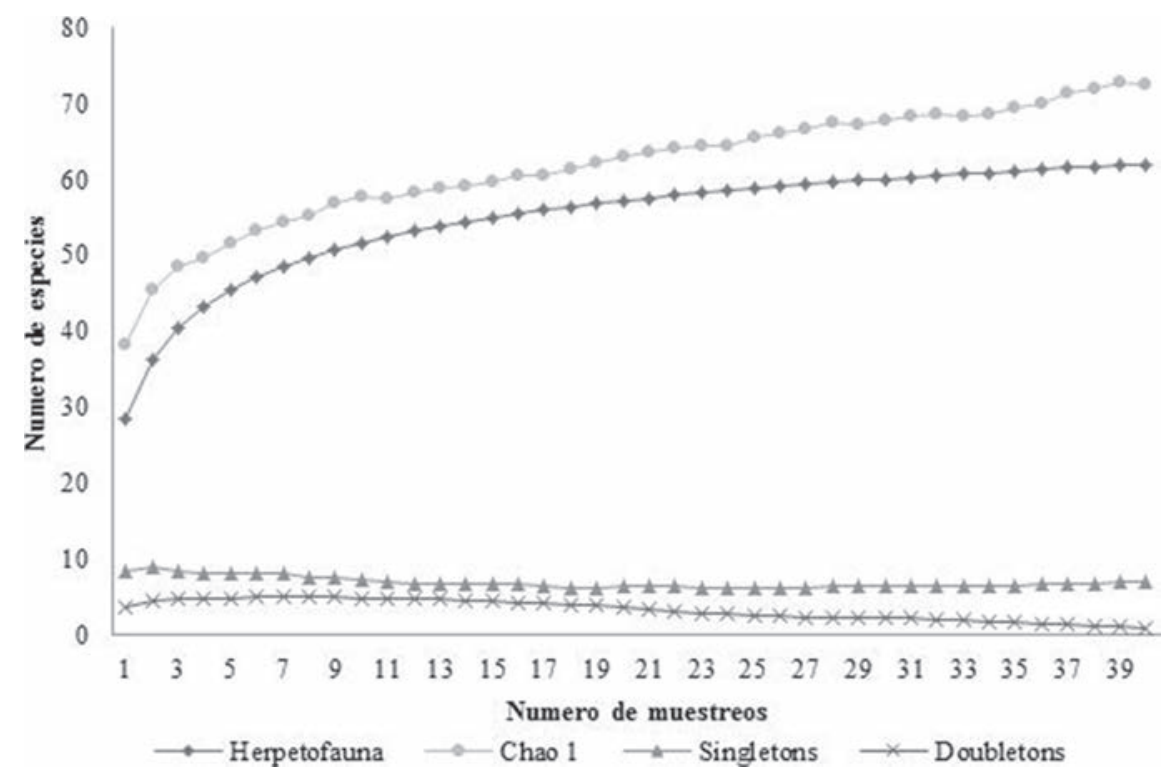

Figura 2. Curvas de acumulación de especies de anfibios y reptiles en cinco coberturas de la tierra en el caserío Casa Aluminio, Dibulla, La Guajira (Colombia). 
Cuadro 2. Abundancia relativa de la herpetofauna registrada en cinco coberturas de la tierra en el caserío Casa Aluminio, Dibulla, La Guajira (Colombia), Cultivo de palma (CP), Mosaico de pastos enmalezados o enrrastrojados con setos de árboles y arbustos (MP), Mosaico de pastos y cultivos transitorios inundables con setos de árboles y arbustos (MPC), Bosque fragmentado inundable con vegetación secundaria (Bfi), Tejido urbano discontinuo (ZU).

\begin{tabular}{|c|c|c|c|c|c|c|c|c|}
\hline Clase & Familia & Especie & Código & $\mathrm{CP}$ & MP & MPC & Bfi & ZU \\
\hline \multirow[t]{17}{*}{ Amphibia } & \multirow[t]{2}{*}{ Bufonidae } & Rhinella granulosa (Spix, 1824) & $\operatorname{Rg}$ & 0.106 & 0.057 & 0.053 & 0.028 & 0.134 \\
\hline & & Rhinella humboldti (Spix, 1824) & $\mathrm{Rh}$ & 0.004 & 0.001 & 0.003 & 0.026 & 0.002 \\
\hline & Ceratophryidae & Ceratophrys calcarata (Boulenger, 1890) & $\mathrm{Cx}$ & & 0.007 & 0.000 & & 0.004 \\
\hline & \multirow[t]{6}{*}{ Hylidae } & Dendropsophus microcephalus (Cope, 1886) & $\mathrm{Dm}$ & 0.062 & 0.118 & 0.173 & 0.002 & 0.012 \\
\hline & & Hypsiboas crepitans ( Wied-Neuwied, 1824) & $\mathrm{Hc}$ & 0.001 & 0.001 & & 0.017 & 0.006 \\
\hline & & Hypsiboas pugnax (Schmidt, 1857) & Hp & 0.030 & 0.022 & 0.011 & 0.060 & 0.030 \\
\hline & & Scarthyla vigilans (Solano, 1971) & $\mathrm{Sv}$ & 0.003 & 0.018 & 0.022 & 0.001 & 0.002 \\
\hline & & Scinax rostratus (Peters, 1863) & $\mathrm{Sr}$ & 0.000 & & & & \\
\hline & & Scinax ruber (Laurenti, 1768) & Sru & & & & & 0.000 \\
\hline & Leiuperidae & Pseudopaludicola pusilla (Ruthven, 1916) & Pp & 0.031 & 0.085 & 0.036 & 0.009 & 0.003 \\
\hline & \multirow[t]{5}{*}{ Leptodactylidae } & Leptodactylus fragilis (Brocchi, 1877) & Lfr & 0.170 & 0.158 & 0.090 & 0.184 & 0.068 \\
\hline & & Leptodactylus fuscus (Schneider, 1799) & Lf & 0.087 & 0.087 & 0.068 & 0.074 & 0.036 \\
\hline & & Leptodactylus insularum (Barbour, 1906) & $\mathrm{Li}$ & 0.125 & 0.139 & 0.242 & 0.007 & 0.219 \\
\hline & & Leptodactylus poecilochilus(Cope, 1862) & Lp & 0.089 & 0.018 & 0.020 & 0.134 & 0.054 \\
\hline & & Leptodactylus cf. Savagei (Heyer, 2005) & Ls & & & 0.000 & & \\
\hline & Microhylidae & Chiasmocleis panamensis (Dunn et al. 1948) & $\mathrm{Cp}$ & & 0.001 & & & 0.003 \\
\hline & Pseudidae & Pseudis paradoxa (Linnaeus, 1758) & Ppx & & 0.001 & & & 0.001 \\
\hline \multirow{12}{*}{ Reptiles } & \multirow{12}{*}{ Colubridae } & Erythrolamprus melanotus (Shaw, 1802) & Em & 0.001 & 0.001 & 0.000 & 0.002 & 0.002 \\
\hline & & Helicops danieli (Amaral, 1938) & $\mathrm{Hd}$ & 0.000 & 0.002 & & & \\
\hline & & Imantodes cenchoa (Linnaeus, 1758) & Ic & 0.000 & & 0.000 & 0.001 & 0.003 \\
\hline & & Leptodeira annulata (Linnaeus, 1758) & Lan & 0.003 & 0.003 & 0.008 & 0.003 & 0.015 \\
\hline & & Leptophis ahaetulla (Linnaeus, 1758) & $\mathrm{La}$ & 0.000 & & 0.001 & 0.001 & 0.003 \\
\hline & & Lygophis lineatus (Linnaeus, 1758) & $\mathrm{Ll}$ & 0.002 & 0.001 & 0.003 & 0.001 & 0.004 \\
\hline & & Mastigodryas boddaerti (Sentzen, 1796) & $\mathrm{Mb}$ & & 0.001 & & & \\
\hline & & Mastigodryas pleei (Dumeril et al. 1854) & $\mathrm{Mp}$ & 0.001 & 0.001 & 0.000 & 0.001 & 0.001 \\
\hline & & Ninia atrata (Hallowell, 1845) & $\mathrm{Na}$ & & & 0.001 & & 0.001 \\
\hline & & Oxybelis aeneus (Wagler, 1824) & $\mathrm{Oa}$ & & 0.001 & 0.001 & & 0.004 \\
\hline & & Pseudoboa neuwiedii (Dumeril et al.1854) & Pn & 0.001 & & & & 0.000 \\
\hline & & Sibon nebulatus (Linnaeus, 1758) & $\mathrm{Sn}$ & & & 0.000 & & 0.002 \\
\hline
\end{tabular}


Cuadro 2. Continúa.

\begin{tabular}{|c|c|c|c|c|c|c|c|c|}
\hline Clase & Familia & Especie & Código & $\mathrm{CP}$ & MP & MPC & Bfi & ZU \\
\hline & & Tantilla melanocephala (Linnaeus, 1758) & $\mathrm{Tm}$ & 0.000 & & 0.000 & 0.001 & 0.001 \\
\hline & & Thamnodynastes gambotensis (Pérez. \& Moreno, 1989) & $\operatorname{Tg}$ & 0.001 & 0.002 & 0.005 & & 0.003 \\
\hline & Emydidae & Trachemys callirostris (Gray, 1856) & Tc & & & 0.000 & 0.001 & \\
\hline & Gekkonidae & Phyllodactylus ventralis (O’Shaughnessy, 1875) & $\mathrm{PV}$ & & & & 0.001 & \\
\hline & & Tretioscincus bifasciatus (Dumeril, 1851) & $\mathrm{Tb}$ & 0.001 & 0.004 & 0.005 & 0.004 & 0.003 \\
\hline & Iguanidae & Iguana iguana (Linnaeus, 1758) & $\mathrm{Ii}$ & 0.001 & 0.001 & 0.002 & & 0.010 \\
\hline & Kinosternidae & Kinosternon scorpioides (Linnaeus, 1766) & Ks & & & 0.000 & & \\
\hline & Polichrotidae & Anolis auratus (Daudin, 1802) & Aau & 0.095 & 0.023 & 0.102 & 0.038 & 0.075 \\
\hline & & Gonatodes vittatus (Lichtenstein, 1856) & Gv & & & 0.000 & 0.064 & 0.001 \\
\hline & & Lepidoblepharis sanctaemartae (Ruthven, 1916) & Lsa & & & & 0.019 & 0.010 \\
\hline & Teiidae & Ameiva praesignis (Baird \& Girard, 1852) & Apr & 0.030 & 0.020 & 0.020 & 0.043 & 0.019 \\
\hline & & Ameiva bifrontata (Cope, 1862) & $\mathrm{Ab}$ & 0.050 & 0.047 & 0.034 & 0.040 & 0.053 \\
\hline & & Cnemidophorus lemniscatus (Linnaeus, 1758) & $\mathrm{Cl}$ & 0.091 & 0.062 & 0.060 & 0.104 & 0.086 \\
\hline & & Holcosus festivus (Lichtenstein, 1856) & Hf & & & 0.000 & & 0.001 \\
\hline & & Tupinambis teguixin (Linnaeus, 1758) & $\mathrm{Tt}$ & 0.000 & & & 0.004 & 0.000 \\
\hline & Viperidae & Porthidium lansbergii (Schlegel, 1851) & $\mathrm{Pl}$ & & & & & 0.001 \\
\hline
\end{tabular}

Cuadro 3. Análisis de abundancia proporcional de la herpetofauna registrada en cinco coberturas de la tierra en el caserío Casa Aluminio, Dibulla, La Guajira (Colombia). Cultivo de palma (CP), Mosaico de pastos enmalezados o enrastrojados con setos de árboles y arbustos (MP), Mosaico de pastos y cultivos transitorios inundables con setos de árboles y arbustos (MPC), Bosque fragmentado inundable con vegetación secundaria (Bfi), Tejido urbano discontinuo (ZU).

\begin{tabular}{ccccc}
\hline Cobertura & $\begin{array}{c}\text { No. Especies } \\
(S)\end{array}$ & $\begin{array}{c}\text { Shannon-Wiener } \\
\left(H^{\prime}\right)\end{array}$ & $\begin{array}{c}\text { Diversidad máxima } \\
\left(H^{\prime} \text { max }\right)\end{array}$ & Pielou \\
$\left(J^{\prime}\right)$ & & & 3.66 & 0.68 \\
CP & 39 & 2.52 & 3.61 & 0.73 \\
MP & 37 & 2.66 & 3.73 & 0.66 \\
MPC & 42 & 2.48 & 3.66 & 0.76 \\
Bfi & 39 & 2.80 & 3.98 & 0.71 \\
ZU & 54 & 2.83 & & \\
\hline
\end{tabular}

fueron en CP, A. auratus (0.094), C. lemniscatus (0.090) y A. bifrontata (0.049); en Bfi, C. lemniscatus (0.103), Gonatodes vittatus (0.064) y Ameiva praesignis (0.043); en MPC, $A$. auratus (0.101), C. lemniscatus (0.06) y A. bifrontata (0.034); en MP, C. lemniscatus (0.061), A. bifrontata (0.047), A. auratus (0.022) y para ZU, C. lemniscatus (0.085), A. auratus (0.074) y A. bifrontata (0.052), siendo ésta de igual manera la cobertura de la tierra que registró mayor riqueza de especies (33) durante el estudio (Cuadro 2).

Las gráficas de rango-abundancia para anfibios y reptiles en cada cobertura de la tierra muestran baja equidad, las curvas presentan una pendiente similar para cada cobertura de la tierra estudiada (Fig. 3; Cuadro 2). 

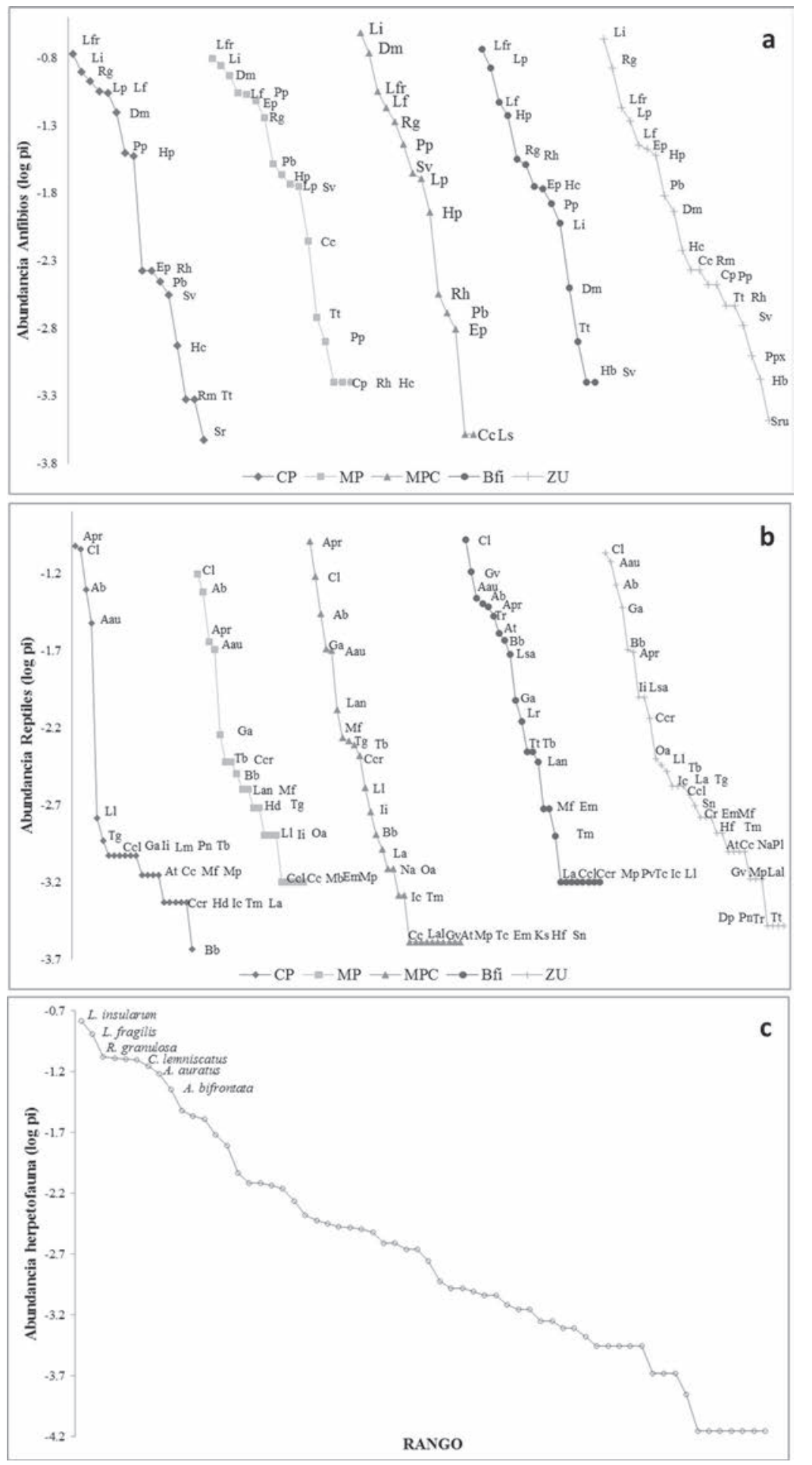

Figura 3. Curvas rango abundancia de la herpetofauna en cinco coberturas de la tierra en el caserío Casa Aluminio, Dibulla, La Guajira (Colombia). (a) Curva de rango abundancia de anfibios registrada por coberturas de la tierra, (b) Curva de rango abundancia de reptiles registrada por coberturas de la tierra, (c) Curva de rango abundancia de la herpetofauna registrada durante el estudio (solamente se muestran los nombres de las especies más abundantes). Cultivo de palma (CP), Mosaico de pastos enmalezados o enrastrojados con setos de árboles y arbustos (MP), Mosaico de pastos y cultivos transitorios inundables con setos de árboles y arbustos (MPC), Bosque fragmentado inundable con vegetación secundaria (Bfi), Tejido urbano discontinuo (ZU). El código para las especies se enlista en el cuadro 2. 


\section{Diversidad beta}

Similitud. El coeficiente de similitud de Jaccard expresa que las coberturas de la tierra que presentaron mayor relación fue la asociación CP y MP $\left(I_{J}=0.68\right)$, mientras que la relación más baja la presentó la asociación Bfi y MP $\left(I_{J}=0.55\right)$. El análisis de similitud de la composición de especies entre las coberturas de la tierra muestra que comparten aproximadamente la mitad de las especies registradas (Cuadro 4).

Complementariedad. El análisis de complementariedad entre los diferentes pares de coberturas de la tierra muestra que éstas son complementarias en porcentajes entre 31 y 44\%, el mayor porcentaje lo presentan las coberturas Bfi y MP (44.8\%) y el menor las coberturas CP y MP (31.1\%) (Cuadro 4).

\section{DISCUSIÓN}

El presente estudio es el primer aporte referente a la composición de la herpetofauna para esta localidad; la riqueza de anfibios y reptiles puede considerarse como alta en relación con otros estudios realizados en el departamento de la Guajira (Blanco-Torres et al. 2013; Hernández-Ruz et al. 2001), ya que presenta igual o mayor número de especies con un esfuerzo de captura, extensión del área de estudio y rango altitudinal menores.

Los registros de anfibios y reptiles encontrados se relacionan con la herpetofauna reportada para la región Caribe y el Bosque seco en Colombia (Sánchez et al. 1995;
Acosta-Galvis 2000; Urbina-Cardona et al. 2014), representando entre el 31 y 55.4\% de las especies registradas (Galeano et al. 2006; Páez et al. 2006; Urbina-Cardona et al. 2014).

Aunque el esfuerzo de captura permitió registrar el $85.51 \%$ de las especies esperadas, se evidencia la necesidad de aumentar los monitoreos en el área de estudio y enriquecer los registros de anfibios y reptiles en particular los referentes a especies raras y crípticas.

Abundancia relativa. Los anfibios y reptiles que presentaron mayor abundancia en cada cobertura, presentan un amplio rango de distribución y pueden desarrollarse en ambientes con diferentes grados de perturbación, son especies consideradas generalistas, Ameiva bifrontata (Harvey et al. 2012), Anolis auratus (Calderón-Espinosa \& Barragán 2014), Cnemidophorus lemniscatus (Figueras et al. 2008; Montgomery et al. 2011), Dendropsophus microcephalus (Bolaños et al. 2008; Bolívar et al. 2009), Leptodactylus insularum (Solís et al. 2008b; Heyer \& de Sá 2011; Heyer \& Heyer 2013), Leptodactylus fragilis (Heyer et al. 2010; González-Duran et al. 2011; Méndez-Narváez et al. 2014), Rhinella granulosa (Narvaes \& Rodrigues 2009; Silvano et al. 2010; Cassemiro et al. 2012).

La relación de las abundancias de anfibios y reptiles en cada cobertura de la tierra se conservan durante el estudio y difieren poco entre éstas, se observa que los registros de mayor composición y abundancia de especies no guardan relación con el tamaño del área de la cobertura, pero sí con la estructura de la cobertura y la influencia de coberturas circundantes, contrario a lo manifestado en la teoría

Cuadro 4. Análisis de diversidad beta (similitud y complementariedad) de la herpetofauna asociada a cinco coberturas de la tierra, registrada en el caserío Casa Aluminio, Dibulla, La Guajira (Colombia). Cultivo de palma (CP), Mosaico de pastos enmalezados o enrrastrojados con setos de árboles y arbustos (MP), Mosaico de pastos y cultivos transitorios inundables con setos de árboles y arbustos (MPC), Bosque fragmentado inundable con vegetación secundaria (Bfi), Tejido urbano discontinuo (ZU), Número de especies en el sitio A (a), Número de especies en el sitio B (b), Número de especies comunes entre los sitios A y B (c), Índice de Jaccard $\left(\mathrm{I}_{\mathrm{J}}\right)$, Riqueza total entre dos coberturas ( $\mathrm{S}_{\mathrm{AB}}$ ), Especies únicas entre dos coberturas $\left(\mathrm{U}_{\mathrm{AB}}\right)$, Complementariedad entre dos coberturas $\left(\mathrm{C}_{\mathrm{AB}}\right)$.

\begin{tabular}{lccccccc}
\hline Relación & $\mathrm{a}$ & $\mathrm{b}$ & $\mathrm{c}$ & $\mathrm{I}_{\mathrm{J}}$ & $\mathrm{S}_{\mathrm{AB}}$ & $\mathrm{U}_{\mathrm{AB}}$ & $\mathrm{C}_{\mathrm{AB}}$ \\
\hline Bfi-CP & 39 & 39 & 31 & 0.660 & 47 & 16 & 0.340 \\
Bfi-MPC & 39 & 42 & 30 & 0.588 & 51 & 21 & 0.412 \\
Bfi-MP & 39 & 37 & 27 & 0.551 & 49 & 22 & 0.449 \\
Bfi-ZU & 39 & 54 & 36 & 0.632 & 57 & 21 & 0.368 \\
CP-MPC & 39 & 42 & 32 & 0.653 & 49 & 17 & 0.347 \\
CP-MP & 39 & 37 & 31 & 0.689 & 45 & 14 & 0.311 \\
CP-ZU & 39 & 54 & 37 & 0.661 & 56 & 19 & 0.339 \\
MPC-MP & 42 & 37 & 30 & 0.612 & 49 & 19 & 0.388 \\
MPC-ZU & 42 & 54 & 38 & 0.655 & 58 & 20 & 0.345 \\
\hline
\end{tabular}


de biogeografía de islas (MacArthur \& Wilson 1967).

Los resultados del presente estudio son similares a lo encontrado en estudios realizados para diferentes grupos zoológicos, donde se registra que algunas relaciones macroecológicas entre parcelas urbanizadas y no urbanizadas se conservan y presentan pocas diferencias principalmente entre área-especies, abundancia-especies y biomasa-especies (Díaz et al. 2007; Galindo-González 2007; Gardner et al. 2007b; Pautasso et al. 2011), y donde se observa que las especies responden a la fragmentación y destrucción de hábitat de forma diferente, favoreciendo o no, a algunas especies, independientemente del tamaño del área de la cobertura de estudio (Gardner et al. 2007b; Mendenhall et al. 2014).

Si se tiene en cuenta el continuo cambio antropogénico en las diferentes coberturas de la tierra con fines agrícolas y ganaderos, además de los cambios e influencia que éstos generan en las ofertas y servicios de cada cobertura, se observa que la distribución de las especies de anfibios presentan algunas diferencias en la composición y abundancias, lo que puede estar relacionado con la sensibilidad del grupo a los cambios generados en el hábitat, que se relacionan directamente con cambios en las ofertas y condiciones ambientales para el desarrollo de algunas especies (Becker et al. 2010; Cáceres-Andrade \& UrbinaCardona 2009).

Los reptiles presentaron igual composición, con diferencias en la distribución de las especies más abundantes, lo que se puede relacionar con lo encontrado por Carvajal-Cogollo \& Urbina-Cardona (2008) para reptiles en fragmentos de bosque seco tropical, en el que no se encontraron diferencias significativas entre las abundancias y composición de especies y se proponen como hipótesis explicativas que: "los reptiles no responden directamente a la pérdida de hábitat (en relación con el tamaño del fragmento)" y "el tamaño de los fragmentos es muy pequeño que no logra excluir el efecto borde, predominando las especies tolerantes al disturbio".

La cobertura Bfi, es diferente en su uso y estructura, presenta una composición y distribución diferente de las especies de anfibios y reptiles abundantes (Leptodactylus fuscus, Leptodactylus poecilochilus, Ameiva praesignis, Gonatodes vittatus), aunque éstas igualmente son consideradas generalistas, oportunistas, de amplio rango de distribución, tolerantes a diferentes hábitat logrando desarrollarse en ambientes modificados (Sartorius et al. 1999; González et al. 2004; Reynolds 2004; Solís et al. 2008a; Harvey et al. 2012; Sugai et al. 2012; Meilink et al. 2013; de Sá et al. 2014).
La cobertura Bfi presenta una composición de anfibios y reptiles generalistas, que se comparten entre coberturas, lo que se relaciona aparentemente con la influencia de las coberturas circundantes, las cuales ejercen mayor presión por perturbación antropogénica, condicionando la dinámica de las especies (Carvajal-Cogollo \& Urbina-Cardona 2008) y favoreciendo a especies tolerantes al disturbio (Suazo-Ortuño et al. 2008).

Diversidad alfa. Las Zonas urbanizadas y los fragmentos de bosque seco tropical fueron las coberturas que presentaron mayor diversidad, y se relacionan entre sí, en cuanto al comportamiento de la diversidad a lo largo de este estudio. Esto puede ser explicado con base en que estas coberturas comparten características en su estructura y distribución, no guardan un patrón de uniformidad en la distribución horizontal y vertical de la cobertura, presentan un sustrato de hojarasca y la mayor distribución vertical, condiciones que brindan mayores ofertas ambientales para el desarrollo de algunas especies de anfibios y reptiles, o bien, la relación de estas coberturas responde a que la cobertura de Bfi, se encuentra fuertemente influenciada por las coberturas circundantes, comportándose como un borde, producto de la transformación antropogénica del ecosistema (Urbina-Cardona \& Reynoso 2005; CarvajalCogollo \& Urbina-Cardona 2008).

Las coberturas CP y MPC presentan una diversidad similar entre sí, estas coberturas comparten características en relación con su distribución horizontal y vertical. Las superficies presentan cubierta de vegetación con alturas no superiores a los $5 \mathrm{~m}$, hay canales de riego que se convierten en una oferta hídrica permanente de estas coberturas, y están sujetas a procesos de inundación en el periodo de lluvias mayores, que las caracterizan como coberturas de suelos húmedos e inundables, lo cual las diferencia de las otras coberturas de la tierra, principalmente de la cobertura MP.

La cobertura de la tierra MP es la cobertura que presentó, a lo largo del estudio, la menor diversidad, lo cual puede deberse a que esta es la cobertura menos compleja, la que presenta una distribución horizontal y vertical menor en su interior, lo que se traduce como la disminución de ofertas de hábitat y ofertas ambientales para el desarrollo de las especies (Urbina-Cardona \& Reynoso 2005; Urbina-Cardona et al. 2014).

Los efectos de la deforestación y la transformación del bosque seco tropical en coberturas de uso agrícola y ganadero, brindan menos ofertas para el desarrollo de las especies, contribuyendo a la disminución de la diversidad y la cobertura vegetal del suelo (García-Oliva \& Jaramillo 2011). 
Los valores de equidad para cada cobertura de la tierra no fueron altos, no se presentan especies con alta dominancia en relación con las demás en cada cobertura, las especies de anfibios y reptiles presentan una variada distribución de las abundancias que responde aparentemente a las características generales y específicas de cada cobertura de la tierra y su uso, que favorecen o no a las especies y sus poblaciones (Gardner et al. 2007b; Suazo-Ortuño et al. 2008; Mendenhall et al. 2014).

Diversidad beta. Los valores de complementariedad de anfibios y reptiles entre las diferentes coberturas de la tierra no son altos, guardan una relación de entre 31 y $44 \%$ de las especies registradas para el área de estudio, y valores de similitud en la composición de especies entre coberturas de 55 y $68 \%$ según el índice de Jaccard, esto puede explicarse en que la estructura vegetal en cada cobertura de la tierra guarda relación con los usos del suelo, aunque cada cobertura presenta características estructurales particulares.

Se registran diferencias en la distribución horizontal y vertical de cada cobertura, representada en mayores o menores oferta de hábitat, que influyen en la estructura y composición de anfibios y reptiles que pueden explicar la complementariedad entre coberturas y se relaciona con lo manifestado por diferentes autores sobre la influencia de la deforestación sobre la riqueza de especies de manera positiva o negativa (Urbina-Cardona et al. 2006; Becker et al. 2009; Reading et al. 2010; Urbina-Cardona et al. 2014).

Se registra que 25 de las 62 especies presentadas en el estudio se encuentran en todas las coberturas de la tierra y éstas en su mayoría coinciden con ser especies generalistas, que presentan amplio rango de distribución y toleran cambios en la estructura del hábitat, lo que se relaciona directamente con las coberturas de la tierra estudiadas y los usos antropogénicos a que están sujetas.

Se desconoce el efecto del borde sobre cada cobertura de la tierra estudiada que puede ser considerado como un factor de influencia en la estructura y composición de anfibios y reptiles (Schlaepfer \& Gavin 2001; CarvajalCogollo \& Urbina-Cardona 2008).

La estructura y distribución horizontal y vertical similar y la distancia cercana entre coberturas pueden contribuir a que la composición de anfibios y reptiles guarden relación, aunque también ésta puede ser el resultado del remplazo gradual de la biota nativa por especies con amplio rango de distribución que presentan mayor tolerancia a los cambios de la cobertura de la tierra y sus usos antropogénicos (McKinney \& Lockwood 1999; Olden et al 2004; McKinney 2006; Olden et al. 2006), los cuales pueden ser difíciles de determinar con el presente estudio, debido a que en la región Caribe los cambios en el bosque seco tropical por labores agrícolas se han realizado durante siglos y los registros históricos de la biota en la localidad de estudio son escasos e incipientes.

Implicaciones para la conservación. El registro de 62 especies de anfibios y reptiles en cinco áreas con diferente grado de perturbación en área rural del municipio de Dibulla, La Guajira, puede interpretarse como un registro de alta diversidad biológica, para este grupo en esta región, más allá del registro de especies, se evidencia la necesidad de estudios más frecuentes que busquen evaluar la situación de la biodiversidad en esta localidad, que permitan interpretar el estado de los fragmentos de Bs-t, su estructura, composición y diversidad funcional asociada, cómo están siendo afectados por las modificaciones de este ecosistema con fines productivos y cómo los cambios en la cobertura de la tierra y los usos antropogénicos del suelo están influyendo en la dinámica espacial, temporal y poblacional de las especies nativas del bosque.

Los patrones de distribución de las especies van más allá del tamaño del área de estudio, algunas relaciones macroecológicas, ayudan a interpretar el comportamiento de la diversidad biológica asociada a diferentes grados de perturbación, donde no se puede calificar una cobertura y uso de la tierra con un comportamiento e impacto igual a otra (Kowarik 2011).

La alta frecuencia de encuentro de especies consideradas generalistas y el alto grado de similitud en la composición de anfibios y reptiles entre coberturas, muestran aparentemente una diversidad homogénea, que puede ser respuesta a la presión natural y antropogénica, histórica, ejercida para esta localidad y otros fragmentos de bosque seco en las tierras bajas del Caribe (Meisel \& Pérez 2006; Urbina-Cardona et al. 2014), condición que evidencia la falta y necesidad de conocimiento base sobre la diversidad biológica en la región y los efectos de la fragmentación sobre ésta.

Para tomar medidas que favorezcan la conservación y recuperación de la biodiversidad, es necesario caracterizar la biota asociada a las diferentes coberturas de la tierra, crear el conocimiento base para desarrollar estudios que permitan interpretar el impacto de los usos del suelo sobre las coberturas de la tierra y la diversidad asociada, para así evidenciar problemáticas más complejas como pérdida de diversidad funcional y homogeneización biótica en la región (Olden et al. 2004; McKinney 2006; Olden et al. 2006; Marchetti et al. 2006; McKinney 2008). 
AGRADECIMIENTOS. A quien por todo fue hecho, a la comunidad del caserío Casa Aluminio, al personal de la finca Las Palmas, en especial a los señores Juvenal y Eliecer por su acompañamiento y apoyo durante el estudio, a la Universidad de la Guajira por su preocupación por el conocimiento de la diversidad biológica del departamento y la región Caribe, de manera especial al personal del centro de investigaciones y de la Facultad de Ciencias Básicas y Aplicadas, programa de Biología, por su colaboración y apoyo durante este proceso de estudios; a los compañeros, amigos y colegas, Bienvenido Bastidas, Yoelis Yepes, Estephani Pimienta y Norelvis Vargas, por su compañía invaluable en campo, a Nicole, Julio y Paola por el tiempo prestado para este estudio.

\section{LITERATURA CITADA}

Acosta-Galvis, A. R. 2015. Lista de los Anfibios de Colombia: Referencia en línea V.05.2015.0 http://www.batrachia.com; Batrachia, Villa de Leyva, Boyacá, Colombia.

Acosta-Galvis, A. R. 2000. Ranas, Salamandras y Caecilias (Tetrapoda: Amphibia) de Colombia. Biota Colombiana, 1: 289-319.

Almeida-Gomes, M. \& Rocha, C. F. D. 2014. Landscape connectivity may explain anuran species distribution in an Atlantic forest fragmented area. Landscape Ecology, 29: 29-40.

Becker, C. G., Fonseca, C. R., Haddad, C. F. B. \& Prado, C. I. 2009. Habitat Split as a Cause of Local Population Declines of Amphibians with Aquatic Larvae. Conservation Biology, 24: 287294.

Becker, C. G., Loyola, R. D., Haddad, C. F. B. \& Zamudio, K. R. 2010. Integrating species life-history traits and patterns of deforestation in amphibian conservation planning. Diversity and Distributions, 16: 10-19.

Bolaños, F., Santos-Barrera, G., Solís, F., Ibáñez, R., Wilson, L.D., Savage, J., Lee, J., Trefaut Rodrigues, M., Caramaschi, U., Mijares, A. \& Hardy, J. 2008. Dendropsophus microcephalus. The IUCN Red List of Threatened Species 2008: e.T55558A11318242. http://dx.doi.org/10.2305/IUCN.UK.2008. RLTS.T55558A11318242.en. (accessed on December 2013).

Bolívar-G., W. Ospina-Sarria, J. J., Méndez-Narváez, J. \& Burbano-Yandi, C. E. 2009. Amphibia, Anura, Hylidae, Dendropsophus microcephalus (Boulenger, 1898): Distribution extensions. Check List, 5: 926-928.

Blanco-Torres, A., Báez, L., Patiño-Flores, E. \& Renjifo, J. M. 2013. Herpetofauna del valle medio del río Ranchería, La Guajira, Colombia. Biodiversidad Neotropical, 3: 113-122.

Cáceres-Andrade, S. P. \& Urbina-Cardona, J. N. 2009. Ensamblajes de anuros de sistemas productivos y bosques en el piedemonte llanero, departamento del Meta, Colombia. Caldasia, 31:175-194.

Calderón-Espinosa, M. L. \& Barragán-Contreras, L. A. 2014. Geographic body size and shape variation in a mainland Anolis (Squamata: Dactyloidae) from Northwestern South America (Colombia). Acta Biológica Colombiana, 19: 167-174.

Campbell, J. A. \& Lamar, W. W. 1989. The venomous reptiles of Latinoamerica. University Press, New York, 425 pp.

Carvajal-Cogollo, J. E. \& Urbina-Cardona, J. N. 2008. Patrones de diversidad y composición de reptiles en fragmentos de bosque se- co tropical en Córdoba, Colombia. Tropical Conservation Science, 1: 397-416.

Cassemiro, F. A. S., Gouveia, S. F. \& Diniz-Filho, J. A. F. 2012. Distribuição de Rhinella granulosa: integrando envelopes bioclimáticos e respostas ecofisiológicas. Revista da Biologia, 8: 3844.

de Sá, R., Grant, T., Camargo, A., Heyer, W. R., Ponssa, M. L. \& Stanley, E. 2014. Systematics of the Neotropical Genus Leptodactylus Fitzinger, 1826 (Anura: Leptodactylidae): Phylogeny, the Relevance of Non-molecular Evidence, and Species Accounts. South American Journal of Herpetology, 9: 1-128.

Díaz, G. N., Iñiguez, D. L. I. \& Santana, C. E. 2007. Ecología y conservación de la nutria (Lontra longicaudis) en la cuenca baja del río Ayuquila, Jalisco. pp. 166-182. In: G. Sánchez-Rojas \& A. Rojas-Martínez (Eds.). Tópicos en sistemática, biogeografía, ecología y conservación de mamíferos. Instituto de Ciencias básicas e ingeniería, Universidad autónoma del estado de Hidalgo, México, D. F.

Duellman, W. E. \& Trueb, L. 1986. Biology of Amphibians. Johns Hopkins University Press, Baltimore, $670 \mathrm{pp}$.

Figueras, J., González S., L. A., Prieto Arcas, A., Velásquez, J. \& Ferrer, H. 2008. Hábitos alimentarios del lagarto Cnemidophorus lemniscatus (Linnaeus, 1758) (Sauria: Teiidae) en dos zonas Xerofíticas del estado Sucre, Venezuela. Acta Biológica Venezolana, 28(2): 11-22.

Galeano, S. P., Urbina-Cardona, J. N., Rivera, C., M. \& Páez, V. 2006. Los Anfibios de Colombia, diversidad y estado de conocimiento. Pp. 106-118. In: M. E. Chaves \& M. Santamaría (Eds.). Informe nacional sobre el avance en el conocimiento y la información de la Biodiversidad 1998 - 2004, Tomo II. Instituto de investigaciones Biológicas Alexander von Humboldt. Bogotá, D. C.

Galindo-González, J. 2007. Efectos de la fragmentación del paisaje sobre poblaciones de mamíferos; el caso de los murciélagos de los Tuxtlas, Veracruz. pp. 97-114. In: G. Sánchez-Rojas \& A. RojasMartínez (Eds.). Tópicos en sistemática, biogeografía, ecología y conservación de mamíferos. Instituto de Ciencias básicas e ingeniería, Universidad autónoma del estado de Hidalgo, México, D.F.

García-Oliva, F. \& Jaramillo, V. J. 2011. Impact of Anthropogenic Transformation of Seasonally Dry Tropical Forests on Ecosystem Biogeochemical Processes. Pp. 159-172. In: R. Dirzo, H. S. Young, H. A. Mooney \& G. Ceballos (Eds.). Seasonally dry tropical forests: Ecology and conservation. Island Press, Washington, D. C.

Gardner, T. A., Barlow, J. \& Perez, C. A. 2007a. Paradox, presumption and pitfalls in conservation Biology: The importance of habitat change for amphibians and reptiles. Biological Conservation, 138: 166-179.

Gardner, T. A., Ribeiro-Junior, M. A., Barlow, J., Ávila-Pires, T. C., Hoogmoed, M. S. \& Peres, C. A. 2007b. The value of primary, secondary and plantation forest for a Neotropical Herpetofauna. Conservation Biology, 21: 775-787.

Glor, R. E., Flecker, A. S., Benard, M. F. \& Power, A. G. 2001. Lizard diversity and agricultural disturbance in a Caribbean forest landscape. Biodiversity and Conservation, 10: 711-723.

González-Duran, G., Gutiérrez-Cárdenas, P.D.A., \& Escobar-Lasso, S. 2011. Leptodactylus fragilis (Mexican white-lipped frog) diet. Herpetological Review, 42: 583-584. 
González, L., Prieto, A., Molina, C. \& Velásquez, J. 2004. Los reptiles de la península de Araya, estado Sucre, Venezuela. Interciencia, 29: 428-434.

Harvey, M. B., Ugueto, G. N. \& Gutberlet Jr., J. L. 2012. Review of Teiid Morphology with a Revised Taxonomy and Phylogeny of the Teiidae (Lepidosauria: Squamata). Zootaxa, 3459: 1-156.

Hedges, S. B. \& Conn, C. E. 2012. A new skink fauna from Caribbean islands (Squamata, Mabuyidae, Mabuyinae). Zootaxa, 3288: 1-244.

Hernández-Ruz, E. J., Castaño-Mora, O. V. Cárdenas-Arévalo, G. \& Galvis-Peñuela, P. A. 2001. Caracterización preliminar de la comunidad de Reptiles de un sector de la Serranía de Perijá, Colombia. Caldasia, 23: 475-489.

Heyer, R., Acosta-Galvis, A., Mijares, A., Solís, F., Ibáñez, R., Hammerson, G., Savage, J., Wilson, L. D., Bolaños, F., Chaves, G. \& Sunyer, J. 2010. Leptodactylus fragilis. The IUCN Red List of Threatened Species 2010: e.T57127A11587519. http://dx.doi. org/10.2305/IUCN.UK.2010-2.RLTS.T57127A11587519.en. (accessed on December 2013).

Heyer, W. R. \& Heyer, M. M. 2013. Systematics, distribution, and bibliography of the frog Leptodactylus insularum Barbour, 1906 (Amphibia: Leptodactylidae). Proceedings of the Biological society of Washington, 126: 204-233.

Heyer, W.R. \& De Sá, R. 2011. Variation, Systematics, and Relationships of the Leptodactylus bolivianus Complex (Amphibia: Anura: Leptodactylidae). Smithsonian Contributions to Zoology, 635: 1-58.

Heyer, W. R., Donnelly, M. A., McDiarmid, R. W. Hayek, L. C.\& Foster, M. S. 1994. Measuring and monitoring Biological diversity, Standard methods for Amphibians. Smithsonian Institution Press. Washington and London, 364 pp.

Hof, C., Araujo, M. B., Jetz, W. \& Rahbek, C. 2011. Additive threats from pathogens, climate and land-use change for global amphibian diversity. Nature, 480: 516-521.

IDEAM. 2010. Leyenda nacional de coberturas de la tierra. Metodología CORINE Land Cover adaptada para Colombia escala 1:100.000. Instituto de Hidrología, Meteorología y Estudios Ambientales. Bogotá, D. C. 72 pp.

Isaacs, J. \& Urbina-Cardona, J. N. 2011. Anthropogenic disturbance and edge effects on Anuran assemblages inhabiting cloud forest fragment in Colombia. Natureza y Conservação, 9: 39-46.

Kowarik, I. 2011. Novel urban ecosystems, biodiversity, and conservation. Environmental Pollution, 159: 1974-1983.

MacArthur, R. H. \& E. O. Wilson. 1967. The theory of island biogeography. Princeton University Press. Princeton, USA. 224 pp.

Magurran, A. E. 2004. Measuring Biological diversity. Blackwell Science. Oxford, 264 pp.

Marchetti, M. P., Lockwood, J. L. \& Light, T. 2006. Effects of urbanization on California fish diversity, Differentiation, homogenization and the influence of spatial scale. Biological Conservation, 127: 310-318.

Mccraine, J. R. \& Hedges, S. B. 2013. A review of the Cnemidophorus lemniscatus group in Central America (Squamata: Teiidae), with comments on other species in the group. Zootaxa, 3288: 1244.

McDiarmid, R. W., Foster, M. S., Guyer, C., Withfield G., J. \& Chernoff, N. 2012. Reptile Biodiversity, Standard methods for in- ventory and monitoring. University of California Press. Berkeley, Los Angeles and London, 412 pp.

McKinney, M. L. 2006. Urbanization as a major cause of biotic homogenization. Biological Conservation, 127: 247-260.

McKinney, M. L. 2008. Effects of Urbanization on species richness. A review of plants and animals. Urban Ecosystems, 11: 161-176.

McKinney, M. L. \& Lockwood, J. L. 1999. Biotic Homogenization: a few winners replacing many losers in the next mass extinction. Trends in Ecology and Evolution, 14: 450-453.

Meilink, W. R., Clegg, J. R., Mayer, C. J., Sabino P., J., Grasso, D., Stegen, G., Segal, M. \& Kok, P. 2013. Confirmation of the presence of the sphaerodactylid lizard Gonatodes vittatus in Guyana, and an indication of a reproductively active population in Georgetown. Salamandra, 49: 59-62.

Meisel Roca, A. \& Pérez V, G. J. 2006. Geografía física y poblamiento en la costa Caribe Colombiana. Documentos de trabajo sobre Economia Regional, 73: 1-76.

Mendenhall, C. D., Frishkoff, L. O., Santos-Barrera, G., Pacheco, J., Mesfun, E., Mendoza, F., Ehrlich, P. R., Ceballos, G., Daily, G. C. \& Pringle, R. M. 2014. Countryside biogeography of Neotropical reptiles and amphibians. Ecology, 95: 856-870.

Méndez-Narváez, J., Ospina-Sarria, J. J. \& Bolívar-G., W. 2014. Diet and trophic ecology of Leptodactylus fragilis (Leptodactylidae) and Dendropsophus columbianus (Anura: Hylidae) in a disturbed area in southwestern Colombia. Herpetology Notes, 7: 299-305.

Montgomery, C., Boback, S. M., Green, S. W., Paulissen, M. A. \& Walker, J. M. 2011. Cnemidophorus lemniscatus (Squamata: Teiidae) on Cayo Cochino Pequeño, Honduras: extent of island occupancy, natural history, and conservation status. Herpetological Conservation and Biology, 6: 10-24.

Moreno, C. E. 2001. Métodos para medir la biodiversidad. M \& TManuales y Tesis SEA, vol. 1. Zaragoza. 84 pp.

Narvaes, P. \& Rodrigues, M. T. 2009. Taxonomic revision of Rhinella granulose species group (Amphibia, Anura, Bufonidae), with a description of a new species. Arquivos de Zoologia, 40: 1-73.

Olden, J. D., Poff, N. L., Douglas, M. R., Douglas, M. E. \& Fausch, K. D. 2004. Ecological and evolutionary consequences of biotic homogenization. Trends in Ecology and Evolution, 19: 18-24.

Olden, J. D. Poff, N. L. \& McKinney, M. L. 2006. Forecasting faunal and floral homogenization associated with human population geography in North America. Biological Conservation, 127: 261271.

Páez, V., Arredondo, J. C., López, C., Martínez, L. M., Molina, C. \& Restrepo, A. 2006. Reptiles de Colombia, diversidad y estado de conocimiento. pp. 118-131. In: M. E. Chaves \& M. Santamaría (Eds.). Informe nacional sobre el avance en el conocimiento y la información de la Biodiversidad 1998-2004, Tomo II. Instituto de investigaciones Biológicas Alexander von Humboldt. Bogotá, D. C.

Pautasso, M., Böhning-Gaese, K., Clergeau, P., Cueto R., V., Dinetti, M., Fernández-Juricic, E., Kaisanlahti-Jokimäki, M., Jokimäki, J., McKinney, M. L., Sodhi, N. S., Storch, D., Tomialojc, L., Weisberg, P. J., Woinarski, J., Fuller, R. A. \& Cantarello, E. 2011. Global macroecology of bird assemblages in urbanized and semi-natural ecosystems. Global Ecology and Biogeography, 20: 426-436. 
Pizano, C., González-M, R., González, M. F., Castro-Lima, F., López, R., Rodriguez, N., Idárraga-Piedrahíta, A., Vargas, W., Vergara-Varela, H., Castaño-Naranjo, A., Devia, W., Rojas, A., Cuadros, H. \& Toro, J. L. 2014. Las plantas de los bosques secos de Colombia. pp. 49-91. In: C. Pizano \& H. García (Eds.). El bosque seco tropical en Colombia. Instituto de Investigación de Recursos Biológicos Alexander von Humboldt (IAvH). Bogotá D. C., Colombia.

Reading, C.J., Luiselli, L.M., Akani, G. C., Bonnet, X., Amori, G., Ballouard, J. M., Filippi, E. Naulleau, G., Pearson, D. \& Rugiero, L. 2010. Are snake populations in widespread decline?. Biology letters, 6(6): 1-4. doi:10.1098/rsbl.2010.0373.

Reynolds, R., Caramaschi, U., Mijares, A., Acosta-Galvis, A., Heyer, R., Lavilla, E. \& Hardy, J. 2004. Leptodactylus fuscus. The IUCN Red List of Threatened Species 2004: e.T57129A11588348. http://dx.doi.org/10.2305/IUCN.UK.2004. RLTS.T57129A11588348.en. (Accessed on December 2013).

Roze, J. A. 1996. Coral Snakes of the Americas-Biology, Identification, and Venoms. Krieger Publishing Company. Malabar, Florida, $340 \mathrm{pp}$.

Sánchez-C, H., Castaño-M., O. \& Cárdenas-A., G. 1995. Diversidad de los Reptiles en Colombia. pp. 277-326. In: J. O. Rangel-Ch. (Eds.). Colombia Diversidad Biótica I. Instituto de Ciencias Naturales-Universidad Nacional de Colombia-Inderena, Bogotá.

Sartorius S., Vitt, L. J. \& Colli, G. R. 1999. Use of naturally and anthropogenically disturbed habitats in Amazonian rainforest by the teiid lizard Ameiva ameiva. Biological Conservation, 90: 91-101.

Silvano, D., Azevedo-Ramos, C., La Marca, E., Narvaes, P., di Tada, I., Baldo, D., Solís, F., Ibáñez, R., Jaramillo, C., Fuenmayor, Q. \& Hardy, J. 2010. Rhinella granulosa. The IUCN Red List of Threatened Species 2010: e.T54655A11182500. http:// dx.doi.org/10.2305/IUCN.UK.2010-2.RLTS.T54655A11182500. en. (accessed on December 2013).

Solís, F., Ibáñez, R., Chaves, G., Bolaños, F., Savage, J., Jaramillo, C., Fuenmayor, Q., Estupinan, R. \& Mijares, A. 2008a. Leptodactylus poecilochilus. The IUCN Red List of Threatened Species 2008: e.T57158A11577603. http://dx.doi.org/10.2305/IUCN. UK.2008.RLTS.T57158A11577603.en. (accessed on December 2013).

Solís, F., Ibáñez, R., Chaves, G., Savage, J., Jaramillo, C., Fuenmayor, Q., Reynolds, R., Caramaschi, U, Mijares, A., Acosta-Galvis, A., Hardy, J., La Marca, E., Manzanilla, J. \& Bolaños, F. 2008b. Leptodactylus bolivianus. The IUCN Red List of Threatened Species 2008: e.T57114A11582479. http://dx.doi. org/10.2305/IUCN.UK.2008.RLTS.T57114A11582479.en. (accessed on December 2013).
Suazo-Ortuño, I., Alvarado-Díaz, J. \& Martínez-Ramos, M. 2008. Effects of conversion of dry tropical forest to agricultural mosaic on herpetofaunal assemblages. Conservation Biology, 22: 362-374.

Sugai, J. L. M. M., Terra, J. \& Ferreira, V. L. 2012. Diet of Leptodactylus fuscus (Amphibian: Anura: Leptodactylidae) in the Pantanal of Miranda river, Brazil. Biota Neotropical, 12: 99-104.

Ugueto, G. N. \& Harvey, M. B. 2011. Revision of Ameiva ameiva Linnaeus (Squamata: Teiidae) in Venezuela: recognition of four species and status of introduced populations in Southern Florida, USA. Herpetological Monographs, 25: 113-170.

Urbina-Cardona, J. N., Londoño-Murcia, M. C. \& García-Ávila, D. G. 2008. Dinámica espacio temporal en la diversidad de serpientes en cuatro hábitats con diferente grado de alteración antropogénica en el parque nacional natural Isla Gorgona, Pacifico Colombiano. Caldasia, 30: 479-493.

Urbina-Cardona, J. N., Navas, C. A., González, I., Gómez-Martínez, M. J., Llano-Mejía, J., Medina-Rangel, G. F. \& BlancoTorres, A. 2014. Determinantes de la distribución de los Anfibios en el bosque seco tropical de Colombia: Herramientas para su conservación. pp. 167-193; 344-345. In: C. Pizano, \& H. García (Eds.). El bosque seco tropical en Colombia. Instituto de Investigación de Recursos Biológicos Alexander von Humboldt (IAvH). Bogotá D. C., Colombia.

Urbina-Cardona, J.N., Olivares-Pérez, M. \& Reynoso, V. H. 2006. Herpetofauna diversity and microenvironment correlates across a pasture-edge-interior ecotone in Tropical rain forest fragments in the Los Tuxtlas Biosphere reserve of Veracruz, Mexico. Biological Conservation, 132: 61-75.

Urbina-Cardona, J. N. \& Reynoso, V. H. 2005. Recambio de anfibios y reptiles en el gradiente potrero-borde-interior en los Tuxtlas, Veracruz, México. Pp. 191-207. In: G. Halffter, J. Soberón, P. Koleff \& A. Melic (Eds.). Sobre Diversidad Biológica: El significado de las Diversidades Alfa, Beta y Gamma. Zaragoza, España.

Whiles, M. R., Lips, K. R., Pringle, C. M., Kilham, S. S., Bixby, R. J., Brenes, R., Connelly, S., Colon-Graud, J., Hunte-Brown, M., Huryn, A. D., Montgomery, Ch. \& Petterson, S. 2006. The effects of amphibian population declines on the structure and function of Neotropical stream ecosystems. Frontiers in Ecology and the Enviroment, 4: 27-34.

Zank, C., Becker, F. G., Abadiel, M., Baldo, D., Maneyro, R. \& Borges-Martins, M. 2014. Climate change and the distribution of Neotropical Red-Bellied toads (Melanophryniscus, Anura, Amphibia): How to prioritize species and populations? PLOS ONE, 9: $1-11$

Zar, J. H. 2010. Biostatistical analysis. Prentice Hall. Upper Saddle River, New Jersey, 944 pp. 